\title{
Aetiological characteristics of adult acute diarrhoea in a general hospital of Shanghai
}

\author{
X. ZHAO*, B. NI, Y. WANG, X. SHEN, C. ZHANG, J. LIU ANd S. LI \\ Microbiology Laboratory, Centre for Disease Control and Prevention of Xuhui, Xuhui District, Shanghai, China
}

Received 8 April 2016; Final revision 27 August 2016; Accepted 7 October 2016;

first published online 3 November 2016

\section{SUMMARY}

Epidemic surveillance is an effective means to determine the characteristics of acute diarrhoea and the benefits of disease control and prevention. The epidemiological, clinical, and aetiological data of adult (aged $\geqslant 15$ years) acute diarrhoea in a general hospital in Shanghai were collected and analysed. Out of 2430 acute diarrhoea patients, 162 subjects were sampled (sample ratio 15:1). The sampled subjects had an average age ( \pm s.D.) of $44 \pm 18$ years; $142(87.7 \%)$ had a history that indicated ingestion of contaminated food; and $40(24 \cdot 7 \%), 54(33 \cdot 3 \%)$, and $73(45 \cdot 1 \%)$ patients had diarrhoea that was attributed to viral, bacterial, and unknown aetiological origins respectively. Viral diarrhoea is mainly prevalent during the winter and spring months, while bacterial and diarrhoea of unknown aetiology occur mainly in the summer months. The average age of the unknown aetiology group (48 \pm 19 years) was significantly older than that of the viral diarrhoea group ( $39 \pm 16$ years). The number of patients with vomiting in the viral group $(30 \cdot 6 \%)$ was significantly higher than that in the bacterial $(17 \cdot 1 \%)$ and unknown aetiology $(8 \cdot 2 \%)$ groups. Viral and bacterial infections are the main cause of acute diarrhoea in Shanghai. However, further effective technological means are needed to improve the surveillance, control, and prevention of acute diarrhoea.

Key words: Acute diarrhoea, acute gastroenteritis, bacterial infection, surveillance, viral infection.

\section{INTRODUCTION}

Acute diarrhoea is a common pathological condition for people of all ages and manifests as an increased frequency of bowel movements and a decrease in the control of defecation [1]. Various aetiological factors cause the reversal of water and electrolyte absorption to secretion in the small and large intestines, leading to acute diarrhoea [2]. In clinical practice, acute diarrhoea is a major symptom of acute gastroenteritis (AG) (inflammation of the stomach, small intestine, or large intestine), leading to a combination of nausea,

\footnotetext{
* Author for correspondence: X. Zhao, MPH, Microbiology Laboratory, Centre for Disease Control and Prevention of Xuhui, 50 Yongchuan Road, Xuhui District, Shanghai 200237, China. (Email: 18918830193@189.cn)
}

vomiting, abdominal pain, cramping, fever, and diarrhoea [2]. Data from the National Centre for Health Statistics of the United States demonstrated that deaths from all-cause gastroenteritis increased from $\sim 7000$ to $>17000$ cases/year from 1999 to 2007 [3]. Although accurate epidemiological data of acute diarrhoea are not available from other countries, AG is highly prevalent in developing areas of the world [1-3].

Infection is the most well-known aetiological cause of AG $[2,4]$, and the rates of AG cases caused by viral, bacterial, and parasitic infections are $50-70 \%, 15-20 \%$, and $10-15 \%$, respectively [2]. AG caused by viral infections is dominated by rotavirus and norovirus [5-7]. Rotavirus causes severe dehydrating gastroenteritis that affects young children $[8,9]$, while norovirus is believed to cause most outbreaks of non-bacterial AG in all age groups [10]. Other viruses, such as enteric 
adenovirus types 40 and 41 , astrovirus, coronavirus, some picornaviruses, and hepatitis A and E viruses, have also been shown to cause AG [2,11,12]. Bacteria are the second leading cause of $\mathrm{AG}$, and the most reported bacteria correlated with AG are Shigella, Salmonella, Campylobacter, diarrhoeagenic Escherichia coli, pathogenic Vibrio, Yersinia, and Clostridium difficile [2]. Other aetiologies, such as medications and toxic ingestions, are also common in AG [2].

Types of AG are mainly categorized into travellers' diarrhoea, foodborne AG, and antibiotic-associated diarrhoea [2]. Travellers' diarrhoea is a concept that specifically refers to healthy individuals infected in epidemic areas via travel [2]. Antibiotic-associated diarrhoea is also called $\mathrm{Cl}$. difficile colitis and often occurs in hospitalized patients [13-15]. Although cases of $C l$. difficile colitis are increasing, its clinical severity is relatively lower than the other two types of AG. The pathogenesis of foodborne gastroenteritis is mainly categorized as a pathogenproduced toxin that is incorporated into the food before it is ingested; the pathogen produces the toxin while in the gastrointestinal tract after the contaminated food is consumed, and then microbes invade the bowel wall directly and release factors that cause acute diarrhoea [2]. Whatever the mechanism of pathogenesis, patients with AG routinely manifest with nausea, vomiting, abdominal pain, diarrhoea, fever, headache, weakness, and dehydration $[2,16]$. Although most patients typically recover within 2 weeks, severely altered electrolyte balance and dehydration are the main contributors to death associated with this condition $[1,2,4]$.

Infectious AG is mainly transmitted via the fecaloral route; therefore, the number of cases could be greatly reduced if sanitary conditions were improved [2]. Along with economic development in China, public and individual sanitary conditions have improved greatly, and as one of the contributions of the government, a thorough surveillance of acute diarrhoea was performed throughout China. Although cases of acute diarrhoea are decreasing, little information regarding the epidemiological, clinical, and aetiological characteristics of acute diarrhoea is currently available. In this study, the data from the diarrhoeal sentinel surveillance in Shanghai were collected and analysed.

\section{MATERIALS AND METHODS}

\section{Study population}

The total population of Shanghai is about $24 \cdot 3$ million. There are 338 hospitals distributed in the 16 administrative districts in Shanghai, of the 338 hospitals, 58 are large-scale general hospitals with bed numbers $>1000$. Xuhui District is one of the central districts has a population of 1.08 million. To survey adult diarrhoea in Shanghai, any patient with diarrhoea identified in each unit of the Shanghai hospital system must be reported to the local Centre for Disease Control and Prevention (CDC). There are 16 CDCs local distributed in the 16 administrative districts in Shanghai. The 16 local CDCs will, carry out the diarrhoeal sentinel surveillance, share data between local CDCs, and report data to the upper level CDC. Diarrhoeal sentinel surveillance consists of local CDC and hospitals under the jurisdiction of each local CDC. Sampling rates were set as follows: tertiary hospital, 15:1; secondary hospital, 10:1; and community hospital, 5:1. The stool samples were collected randomly in accordance with the above rates. All samples are sent to the local CDC to complete virus and bacteria detections within the prescribed time limit and in accordance with the requirements of the delivery of biological samples. The Shanghai Jiao Tong University Affiliated Sixth People's Hospital is a general hospital located at the urbanrural fringe of Shanghai, which is mainly responsible for the diagnosis and treatment of intestinal diseases in the southwestern region of Shanghai. From October 2013 to September 2014, there were 2430 acute diarrhoea patients diagnosed with $\mathrm{AG}$ in this hospital, of the 2430 acute diarrhoea patients, stool samples of 162 were included in this study.

\section{Data collection}

Age, gender, profession, initial symptoms, symptoms of AG, history of ingesting potentially contaminated food (expired food and spoiled food) and water (expired bottled water and unboiled water), contact with diarrhoeal patients, travel 1 week before the onset of diarrhoea, and administration of antibiotics were collected by a questionnaire survey.

Watery diarrhoea, bloody diarrhoea, dehydration, fever, heart rate, blood pressure, routine blood examination, and a stool test were checked by the initial clinicians or performed in the clinical laboratory of the hospital.

\section{Virus detection}

Faecal specimens were prepared as a $10 \%(w / v)$ suspension in distilled water and then centrifuged for 
$10 \mathrm{~min}$ at $10000 \mathrm{~g}$. Viral RNA and viral DNA were extracted from the suspensions using QIAamp Viral RNA Mini kit and QIAamp DNA Mini kit (Qiagen, The Netherlands), according to the manufacturer's instructions. The nucleic acid pellet was redissolved in $10 \mu \mathrm{l}$ of $10 \mathrm{mmol} / \mathrm{l}$ dithiothreitol containing $5 \%$ (v/v) RNasin (40 U/ $\mu \mathrm{l}$; Promega, USA) and stored at $-80{ }^{\circ} \mathrm{C}$ until use. The virus panel was established by information from the World Health Organization, the US Centers for Disease Control and Prevention, and our accumulation of routine diarrhoea surveillance data [17-19]. Rotavirus, norovirus, adenovirus, astrovirus, sapovirus, mimiviruses, aichivirus, bocavirus, parechovirus, cytomegalovirus, hepatitis A, coronaviruses, picornaviruses, toroviruses, and other enteroviruses were detected by polymerase chain reaction (PCR) or reverse transcription PCR using primer sets as reported previously [17-20].

\section{Bacteria detection}

Detection of bacteria was carried out via classic isolation, bacterial cultures, and identification in combination with molecular diagnostic strategies. Faecal culture was performed according to the 'Laboratory diagnosis of gastrointestinal and pancreatic disorders' [19]. Molecular diagnosis was performed with bacteria species-specific PCR using primer sets reported previously [17-20]. In addition, all samples were simultaneously submitted to VITEK ${ }^{\circledR} 2$ Compact (bioMérieux, France) for microbial identification.

The above technologies covered the most common diarrhoea-related bacteria including Staphylococcus aureus, Cl. perfringens, Bacillus cereus, enterotoxigenic Escherichia coli, enteropathogenic E. coli, Campylobacter jejuni, Salmonella species, Vibrio cholerae O1 and O139 with the cholera toxin gene, $V$. cholerae $\mathrm{O} 1$ and $\mathrm{O} 139$ without the cholera toxin gene, non-cholerae vibrios and others [20]. The diagnosis of epidemic dysentery and cholera was performed according to the Laboratory Methods for the Diagnosis of Epidemic Dysentery and Cholera recommended by the World Health Organization and the US Centers for Disease Control and Prevention [20].

\section{Statistical analysis}

Continuous variables are presented as means \pm standard deviation (s.D.) and categorical data are presented as the number (percentage). Differences between groups were examined using Fisher's exact probability
Table 1. Summary of patients with diarrhoea $(n=162)$

\begin{tabular}{ll}
\hline \hline Male & $85(52 \cdot 5 \%)$ \\
Age, years & $44 \pm 18$ \\
Symptoms & $103(63 \cdot 6 \%)$ \\
Watery diarrhoea & $42(25 \cdot 9 \%)$ \\
Abdominalgia & $33(20 \cdot 4 \%)$ \\
Nausea & $29(17 \cdot 9 \%)$ \\
Vomiting & $28(17 \cdot 3 \%)$ \\
Fever & \\
Medical history & $142(87 \cdot 7 \%)$ \\
History of ingesting potentially & \\
$\quad$ contaminated food & $0(0 \%)$ \\
History of drinking potentially & \\
$\quad$ contaminated water & $0(0 \%)$ \\
History of contact with diarrhoea patients & \\
Outcome & $5(3 \cdot 1 \%)$ \\
Intravenous infusion & $3(1 \cdot 9 \%)$ \\
Hospitalized patients & $1(0 \cdot 6 \%)$ \\
Dehydration & \\
\hline \hline
\end{tabular}

Continuous variables are presented as means \pm standard deviation and categorical data are presented as number $(\%)$.

test or variance analysis according to the characteristics of data distribution. $P<0.05$ was considered to indicate statistical significance.

\section{Ethical statement}

This study was conducted in accordance with the World Medical Association Declaration of Helsinki and approved by the Internal Review Board at the Centers for Disease Control and Prevention of Shanghai (SCDC, Ethical Approval no.: SCDC 2013002). Written informed consent was obtained according to the guidelines of the National Ethics Regulation Committee. Participants, immediate relatives, caregivers, or legal guardians informed the participants of their right to withdraw consent.

\section{RESULTS}

\section{Patient characteristics}

There were 2430 diarrhoea patients that visited the gastrointestinal clinic of diarrhoea sentinel surveillance from October 2013 to September 2014, and based on a sampling ratio of $1: 15$, data of 162 subjects were included in this report. No deaths caused by AG were reported. As shown in Table 1, of the 162 subjects, $85(52.5 \%)$ were males and the average age was $44 \pm 18$ years. Patients presented with the following symptoms: watery diarrhoea $(103,63.6 \%)$, 
Table 2. Aetiology summary

\begin{tabular}{ll}
\hline \hline Bacteria & $40(24 \cdot 7)$ \\
Salmonella & $13(8 \cdot 0)$ \\
Vibrio parahaemolyticus & $11(6 \cdot 8)$ \\
Enterotoxigenic Escherichia coli (ETEC) & $7(4 \cdot 4)$ \\
Enteropathogenic Escherichia coli (EPEC) & $5(3 \cdot 1)$ \\
Campylobacter jejuni & $4(2 \cdot 5)$ \\
Enteroaggregative Escherichia coli (EAggEC) & $3(1 \cdot 9)$ \\
Salmonella + Vibrio parahaemolyticus & $3(1 \cdot 9)$ \\
Viruses & $54(33 \cdot 3)$ \\
Norovirus GII & $25(15 \cdot 4)$ \\
Rotavirus A & $10(6 \cdot 2)$ \\
Norovirus GI & $8(4 \cdot 9)$ \\
Astrovirus & $7(4 \cdot 3)$ \\
Sapovirus & $4(2 \cdot 5)$ \\
Rotavirus B & $1(0 \cdot 6)$ \\
Norovirus GI + GII & $1(0 \cdot 6)$ \\
Bacteria + viruses & $5(3 \cdot 1)$ \\
EPEC + norovirus GII & $2(1 \cdot 2)$ \\
EPEC + norovirus GI & $1(0 \cdot 6)$ \\
EPEC + sapovirus & $1(0 \cdot 6)$ \\
Salmonella + sapovirus & $1(0 \cdot 6)$ \\
Unknown & $73(45 \cdot 1)$ \\
\hline \hline
\end{tabular}

Categorical data are presented as number (\%).

abdominalgia $(42,25 \cdot 9 \%)$, nausea $(33,20 \cdot 4 \%)$, vomiting $(29,17 \cdot 9 \%)$, and fever $(28,17 \cdot 3 \%)$. In all, 142 $(87.7 \%)$ patients had a history of ingesting potentially contaminated food, and no patient had a history of drinking contaminated water or had a history of contact with diarrhoeal patients. Only five $(3 \cdot 1 \%)$, three $(1.9 \%)$, and one $(0.6 \%)$ patients were treated with intravenous infusion, hospitalized, or showed dehydration, respectively. All patients recovered within 10 days.

\section{Aetiology}

Of the 162 diarrhoeal subjects, $40(24 \cdot 7 \%)$ and 54 $(33.3 \%)$ were identified as viral and bacterial infections, respectively, and five $(3 \cdot 1 \%)$ subjects exhibited co-infection with both viruses and bacteria (Table 2). For bacterial infections, $13(8 \cdot 0 \%), 11(6 \cdot 8 \%)$, seven $(4 \cdot 4 \%)$, five $(3 \cdot 1 \%)$, four $(2 \cdot 5 \%)$, three $(1 \cdot 9 \%)$, and three $(1.9 \%)$ subjects were identified as having Salmonella, $V$. parahaemolyticus, enterotoxigenic E. coli (ETEC), enteropathogenic E. coli (EPEC), $C$. jejuni, enteroaggregative E. coli (EAggEC), and Salmonella $+V$. parahaemolyticus infections, respectively (Table 2). For viral infections, 25 (15.4\%), $10(6 \cdot 2 \%)$, eight $(4 \cdot 9 \%)$, seven $(4 \cdot 3 \%)$, four $(2 \cdot 5 \%)$, one $(0 \cdot 6 \%)$, and one $(0.6 \%)$ subjects were identified as having norovirus GII, rotavirus A, norovirus GI, astrovirus, sapovirus, rotavirus $\mathrm{B}$, and norovirus GI + GII infections, respectively (Table 2). Notably, there were as many as $73(45 \cdot 1 \%)$ subjects that could not receive a definite aetiological diagnosis.

\section{Constituent ratio of identified viruses and bacteria}

As shown in Figure 1, of the identified viruses, 46.3\%, $18 \cdot 5 \%, 14 \cdot 8 \%, 13 \cdot 0 \%, 7 \cdot 4 \%$, and $1 \cdot 8 \%$ were identified as norovirus GII, rotavirus A, norovirus GI, astrovirus, sapovirus and rotavirus $\mathrm{B}$, respectively. Of the identified bacteria, $32 \cdot 5 \%, 27 \cdot 5 \%, 18 \cdot 2 \%, 12 \cdot 5 \%$, $10.0 \%$, and $7.5 \%$ were determined to be Salmonella, $V$. parahaemolyticus, ETEC, EPEC, C. jejuni, and EAggEC, respectively.

\section{Seasonal characteristics of diarrhoea}

For cases of viral diarrhoea, 7, 4, 4, 0, 2, 2, 3, 4, 1, 7, 7, and 13 cases were distributed in January-December, respectively, with peak infection emerging in the winter and spring months (Fig. 2). For bacterial diarrhoea, 2, $0,0,1,2,3,12,9,8,1,1$, and 1 cases were distributed in January-December, respectively, with peak infection emerging in the summer months (Fig. 2). For diarrhoea with an unknown aetiology, 3, $2,2,6,2,2,16,21,13,2,3$, and 1 cases were distributed in January-December, respectively, with peak infection also occurring in the summer months (Fig. 2).

\section{Distribution characteristics of observed indexes in groups}

To determine if there were any differences in the age, gender, symptoms, medical history, and outcomes of patients in the groups, the distribution characteristics of the above indexes were evaluated. As shown in Table 3, there were no significant differences in the three groups regarding male ratio, medical history, and outcome. The average age of the aetiology unknown group (48 \pm 19 years) was significantly higher than that of the viral diarrhoea group (39 \pm 16 years) (Table 3 ). In regard to symptoms, no significant difference was observed in the rates of patients with nausea or watery diarrhoea in the three groups (Table 3). The rate of patients with vomiting in the viral group $(30.6 \%)$ was significantly higher than that of the bacterial $(17 \cdot 1 \%)$ and aetiology unknown $(8 \cdot 2 \%)$ groups (Table 3$)$. Notably, the rates of patients with fever or abdominalgia in the bacterial group had the potential to be significantly higher than that of the 

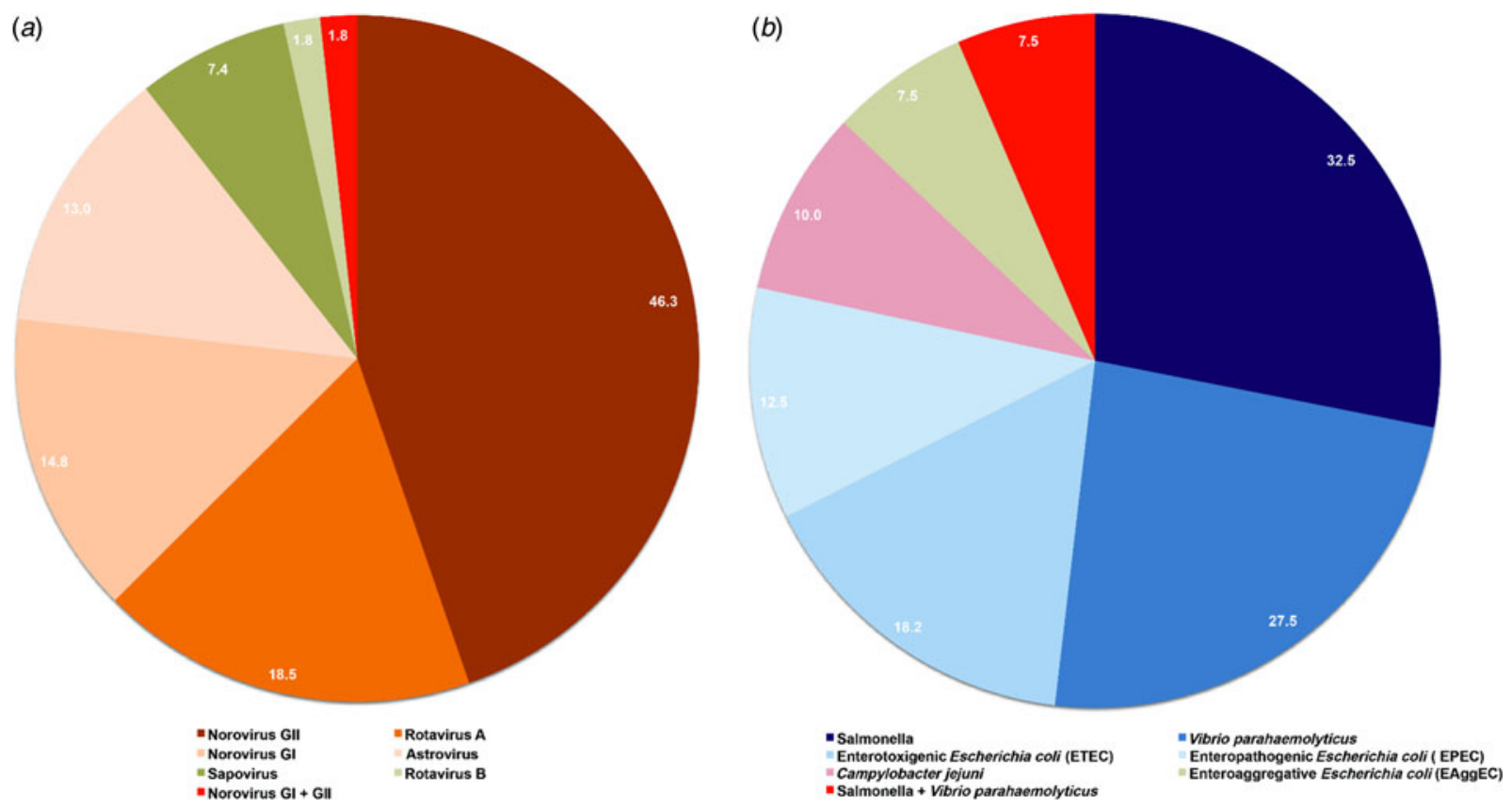

Fig. 1. Constituent ratios of identified $(a)$ viral and $(b)$ bacterial cases. The ratios were calculated as cases of certain viral or bacterial strains identified/total identified viral or bacterial cases. (a) Norovirus GI, norovirus genogroup I cases; norovirus GII, norovirus genogroup II cases; norovirus GI + GII, both norovirus GI and norovirus GI were identified. (b) ETEC, enterotoxigenic Escherichia coli cases; EPEC, enteropathogenic E. coli cases; EAggEC, enteroaggregative E. coli cases.

other two groups if the number of patients was increased (Table 3).

\section{DISCUSSION}

Acute diarrhoea is a common disease that leads to considerable mortality [1-3]. The major pathophysiological mechanism(s) resulting in death are dehydration and a loss of electrolyte balance [21, 22]. Although cases of acute diarrhoea could be significantly reduced if sanitary conditions were improved [1-3], acute diarrhoea or AG is still prevalent in developed countries [1-10]. China is a developing country with a large population that has exhibited a high prevalence of acute diarrhoea in the past [23]. Along with economic development in China, public and individual sanitary conditions have improved greatly and careful monitoring of the presence of acute diarrhoea has been performed. However, scientists outside of China have limited data regarding the epidemiological, clinical, and aetiological characteristics of acute diarrhoea in China. In this report, 162 subjects were randomly recruited from a total of 2430 acute diarrhoea patients who were part of the local annual report based on sentinel surveillance in Shanghai. Overall, no deaths related to acute diarrhoea were reported, and all patients recovered within 10 days. Our main conclusions follow. First, $87.7 \%$ of patients had a history of ingesting potentially contaminated food products, suggesting that foodborne AG is the major type of AG observed in Shanghai. Only $24.7 \%$ and $33.3 \%$ of infections were identified as being caused by viral and bacterial pathogens, respectively. Therefore, a definitive aetiological diagnosis was unable to be made in as many as $45 \cdot 1 \%$ of the subjects, which suggests that more effective technological methods are required to improve the ability to detect the organisms contributing to the development of acute diarrhoea. We also found that viral diarrhoea was more prevalent in the winter and spring months, while diarrhoea caused by bacteria or an unknown aetiology occurred mainly in the summer months. These findings are consistent with other reports and the compiled data from the SCDC [24-26]. In addition, the average age of patients with illness caused by unknown aetiology was significantly higher than that of the viral diarrhoea group, which further emphasizes the importance of more effective pathogen-detection technology. Finally, the number of patients exhibiting vomiting in the viral group was significantly higher than that observed in patients from the bacterial and unknown aetiology groups. 


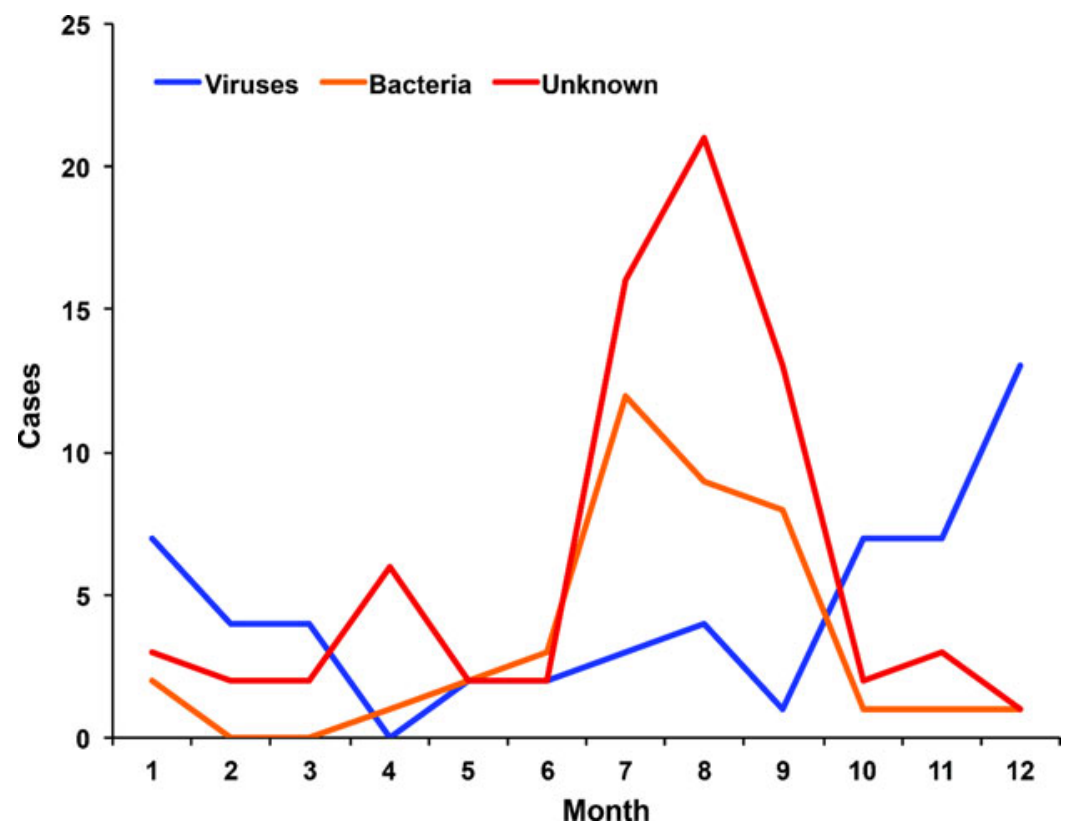

Fig. 2. Seasonal characteristics of diarrhoeal cases. The cases of diarrhoea were calculated per month, and cases of diarrhoea caused by viral, bacterial, and unknown aetiology were calculated independently.

Table 3. Distribution characteristics of observed indexes among diarrhoea patients

\begin{tabular}{lllll}
\hline \hline Index & Viruses $(n=49)$ & Bacteria $(n=35)$ & Unknown $(n=73)$ & $P$ \\
\hline Male & $31(63 \cdot 3 \%)$ & $17(48 \cdot 6 \%)$ & $34(46 \cdot 6 \%)$ & $0 \cdot 173$ \\
Age, years & $39 \pm 16$ & $43 \pm 19$ & $48 \pm 19$ & $0 \cdot 028$ \\
Symptoms & & & & \\
$\quad$ Fever & $7(14 \cdot 3 \%)$ & $11(31 \cdot 4 \%)$ & $10(13 \cdot 7 \%)$ & $0 \cdot 058$ \\
$\quad$ Nausea & $14(28 \cdot 6 \%)$ & $8(22 \cdot 9 \%)$ & $11(15 \cdot 1 \%)$ & $0 \cdot 191$ \\
$\quad$ Abdominalgia & $8(16 \cdot 3 \%)$ & $14(40 \cdot 0 \%)$ & $20(27 \cdot 4 \%)$ & $0 \cdot 053$ \\
$\quad$ Vomiting & $15(30 \cdot 6 \%)$ & $6(17 \cdot 1 \%)$ & $6(8 \cdot 2 \%)$ & $0 \cdot 006$ \\
$\quad 32(65 \cdot 3 \%)$ & $21(60 \cdot 0 \%)$ & $46(63 \cdot 0 \%)$ & $0 \cdot 882$ \\
$\quad$ Watery diarrhoea & & & & \\
Medical history & $44(89 \cdot 8 \%)$ & $29(82 \cdot 9 \%)$ & $65(89 \cdot 0 \%)$ & $0 \cdot 58$ \\
$\quad$ History of ingesting potentially contaminated food & $0(0 \%)$ & $0(0 \%)$ & - \\
$\quad$ History of drinking potentially contaminated water & $0(0 \%)$ & $0(0 \%)$ & $0(0 \%)$ & - \\
$\quad$ History of contact with diarrhoea patients & $0(0 \%)$ & $0(0 \%)$ & $1(1 \cdot 4 \%)$ & - \\
Outcome & $0(0 \%)$ & $0(0 \%)$ & $2(2 \cdot 7 \%)$ & - \\
$\quad \begin{array}{l}\text { Dehydration } \\
\text { Hospitalized patients }\end{array}$ & $1(2 \cdot 0 \%)$ & $0(0 \%)$ & $1(1 \cdot 4 \%)$ & - \\
$\quad$ Intravenous infusion & $0(0 \%)$ & $4(11 \cdot 4 \%)$ & \\
\hline \hline
\end{tabular}

Continuous variables are presented as means \pm standard deviation and categorical data are presented as number (\%). The five patients infected with both viruses and bacteria were not included in this analysis.

Differences between groups were examined by Fisher's exact probability test or variance analysis according to the characteristics of data distribution.

The rates of patients exhibiting a fever or abdominalgia tended to vary by aetiology. Patients with a fever or abdominalgia in the bacterial group had the potential to be significantly higher than that observed in the viral diarrhoea or unknown aetiology groups, if the number of the patients was increased. The differences observed in the symptoms should be the manifestation of viral and bacterial pathogenesis [21, 22, 27]. Current pathogen identification is mainly based on microbe isolation, culture, and nucleic acid testing. However, these detection methods may not lead to a definitive aetiological diagnosis [28]. In this 
study, as many as $45 \cdot 1 \%$ of patients had diarrhoea caused by an unknown aetiology. However, based on the seasonal characteristics observed in cases of diarrhoea with an unknown aetiology, the major cause of disease in this group is likely to be bacterial pathogens given that peak infection also occurred in the summer months. Nevertheless, we were unable to determine the potential impact of toxic ingestions in this population.

For the aetiology of infectious AG, a recent review showed that the proportions of viral, bacterial, and parasitic infections were $50-70 \%, 15-20 \%$, and 10 $15 \%$, respectively [2], which differs from our results. While we did not provide data on the presence of parasites, only $24.7 \%$ and $33.3 \%$ of infections were identified as the result of viral and bacterial pathogens, respectively. The prevalence of viral infections was lower and the occurrence of bacterial infections was higher than what has been reported outside of China, which might represent one of the characteristics of diarrhoeal illness in Shanghai. AG caused by viral infections is dominated by rotavirus and norovirus [5-7]. Our data are in full agreement with this conclusion, and the rates of rotavirus and norovirus were determined to be $18 \cdot 5 \%$ and $61 \cdot 1 \%$, respectively. In addition, astrovirus and sapovirus were also identified in our study population. The most reported bacteria causing AG are Shigella, Salmonella, Campylobacter, diarrhoeagenic E. coli, pathogenic Vibrio, Yersinia, and $\mathrm{Cl}$. difficile [2]. Our data identified the following bacteria, in order of most to least prevalent: Salmonella, V. parahaemolyticus, ETEC, EPEC, C. jejuni, and EAggEC; this is different from a previous report [2] and might represent additional characteristics of diarrhoeal illness in China.

In the present study, the surveillance was focused only on adults; however, we also performed surveillance on diarrhoea in children in other sentinel surveillances in Shanghai, as children have their own epidemiological, clinical, and aetiological characteristics observed in diarrhoeal illness, and these data will be the focus of future reports.

\section{ACKNOWLEDGEMENTS}

There was no specific financial support for this report

\section{DECLARATION OF INTEREST}

None.

\section{REFERENCES}

1. Thielman NM, Guerrant RL. Clinical practice. Acute infectious diarrhoea. New England Journal of Medicine 2004; 350: 38-47.

2. Graves NS. Acute gastroenteritis. Primary Care: Clinics in Office Practice 2013; 40: 727-741.

3. CDC Division of News and Electronic Media. Deaths from gastroenteritis double (http://www.cdc.gov/media/ releases/2012/p0314_gastroenteritis.html). Accessed 30 June 2016.

4. Dickinson B, Surawicz CM. Infectious diarrhoea: an overview. Current Gastroenterology Reports 2014; 16: 399.

5. Matson DO, Estes MK. Impact of rotavirus infection at a large pediatric hospital. Journal of Infectious Diseases 1990; 162: 598-604.

6. Tucker AW, et al. Cost-effectiveness analysis of a rotavirus immunization program for the United States. Journal of the American Medical Association 1998; 279: 1371-1376.

7. Ahmed SM, et al. Global prevalence of norovirus in cases of gastroenteritis: a systematic review and meta-analysis. Lancet Infectious Diseases 2014; 14: 725-730.

8. Grimwood K, Buttery JP. Clinical update: rotavirus gastroenteritis and its prevention. Lancet 2007; 370: 302-304.

9. Parashar UD, et al. Global mortality associated with rotavirus disease among children in 2004. Journal of Infectious Diseases 2009; 200: S9-S15.

10. Getto L, Zeserson E, Breyer M. Vomiting, diarrhoea, constipation, and gastroenteritis. Emergency Medicine Clinics of North America 2011; 29: 211-237.

11. Cao J, et al. Hepatitis A outbreaks in China during 2006: application of molecular epidemiology. Hepatology International 2009; 3: 356-363.

12. Lu J, et al. General epidemiological parameters of viral hepatitis A, B, C, and E in six regions of China: a crosssectional study in 2007. PLoS ONE 2009; 4: e8467.

13. Campbell RR, et al. Clostridium difficile in acute and long-stay elderly patients. Age Ageing 1988; 17: 333 336.

14. Kim J, et al. Epidemiological features of Clostridium difficile-associated disease among inpatients at children's hospitals in the United States, 2001-2006. Pediatrics 2008; 122: 1266-70.

15. McDonald LC, Owings M, Jernigan DB. Clostridium difficile infection in patients discharged from US shortstay hospitals, 1996-2003. Emerging Infectious Diseases 2006; 12: 409-415.

16. Zollner-Schwetz I, Krause R. Therapy of acute gastroenteritis: role of antibiotics. Clinical Microbiology and Infection 2015; 21: 744-749.

17. Platts-Mills JA, Operario DJ, Houpt ER. Molecular diagnosis of diarrhoea: current status and future potential. Current Infectious Disease Reports 2012; 14: 41-46.

18. Soli KW, et al. Detection of enteric viral and bacterial pathogens associated with paediatric diarrhoea in Goroka, Papua New Guinea. International Journal of Infectious Diseases 2014; 27: 54-58. 
19. Salwen MJ, et al. Laboratory diagnosis of gastrointestinal and pancreatic disorders. In: McPherson RA, Pincus MR, eds. Henry's Clinical Diagnosis and Management by Laboratory Methods. Philadelphia: Saunders, 2011, chapter 22.

20. Centers for Disease Control and Prevention. Laboratory methods for the diagnosis of epidemic dysentery and cholera (www.cdc.gov/cholera/pdf/Laboratory-Methodsfor-the-Diagnosis-of-Epidemic-Dysentery-and-Cholera.pdf. Accessed 4 April 2016.

21. Kaper JB, Nataro JP, Mobley HL. Pathogenic Escherichia coli. Nature Reviews Microbiology 2004; 2: 123-140.

22. Tsolis RM, et al. From bench to bedside: stealth of enteroinvasive pathogens. Nature Reviews Microbiology 2008; 6: 883-892.

23. Snyder JD, Merson MH. The magnitude of the global problem of acute diarrheal disease: a review of active surveillance data. Bulletin of the World Health Organization 1982; 60: 605-613.

24. Babji S, et al. Multi-centre surveillance of rotavirus diarrhoea in hospitalized children $\leqslant 5$ years of age in India, 2009-2012. Vaccine 2014; 32: A10-12.

25. Ahmed SM, Lopman BA, Levy K. A systematic review and meta-analysis of the global seasonality of norovirus. PLoS ONE 2013; 8: e75922.

26. Das SK, et al. Gastroenteritis due to typhoidal Salmonella: a decade of observation at an urban and a rural diarrheal disease hospital in Bangladesh. BMC Infectious 2014; 14: 435.

27. Ramig RF. Pathogenesis of Intestinal and Systemic Rotavirus Infection. Journal of Virology 2004; 78: 10213-10220.

28. Guerrant RL, et al. Practice guidelines for the management of infectious diarrhoea. Clinical Infectious Diseases 2001; 32: 331-351. 